\title{
SINGULAR POINTS OF TRANSFORMATIONS AND TWO-PARAMETER FAMILIES OF CURVES.
}

BY DR. W. V. LOVITT.

\section{Introduction.}

In the Transactions for October, 1915, I discussed some singularities of a point transformation in three variables

$$
x=\phi(u, v, w), \quad y=\psi(u, v, w), \quad z=\chi(u, v, w) .
$$

Let a particular one of the singular points in question be denoted by $P$, and let $S$ denote the surface through $P$ in the $u v w$-space defined by setting the jacobian of the transformation equal to zero. The point $P$ and the surface $S$ are transformed by (1) into a point $P_{1}$ and surface $S_{1}$ in the $x y z$-space.

In the present paper there is found on the surface $S_{1}(x, y, z)$ a curve $\left(d_{1}\right)$ which is the envelope of a one-parameter family of curves properly chosen from the two-parameter family (1). We find in the $u v w$-space that plane of directions which transforms into the direction of the curve $\left(d_{1}\right)$ in the $x y z$-space.

\section{Initial Assumptions.}

Let us consider a real point transformation of three-space

$$
x=\phi(u, v, w), \quad y=\psi(u, v, w), \quad z=\chi(u, v, w)
$$

with determinant

$$
J(u, v, w) \equiv\left|\begin{array}{lll}
\phi_{u} & \phi_{v} & \phi_{w} \\
\psi_{u} & \psi_{v} & \psi_{w} \\
\chi_{u} & \chi_{v} & \chi_{w}
\end{array}\right|
$$

The functions $\phi, \psi, \chi$ are not necessarily analytic but it will be presupposed that

(a) the functions $\phi, \psi, \chi$ are of class $C^{\prime \prime \prime} *$ in a neighborhood of the origin $(u, v, w)=(0,0,0)$;

* We shall say that a single-valued function $f$ of $u, v, w$ is of class $C^{\prime \prime \prime}$ if $f(u, v, w)$ and its partial derivatives of orders one, two, and three are continuous in a region in which $f$ is defined. 
(b) the following initial conditions are satisfied:

$$
\phi(0,0,0)=\psi(0,0,0)=\chi(0,0,0)=0 ;
$$

(c) $J(0,0,0)=0$;

(d) at the origin $(u, v, w)=(0,0,0)$ at least one of the determinants of the matrix

is different from zero.

$$
\left\|\begin{array}{lll}
J_{u} & J_{v} & J_{w} \\
\phi_{u} & \phi_{v} & \phi_{w} \\
\psi_{u} & \psi_{v} & \psi_{w} \\
\chi_{u} & \chi_{v} & \chi_{w}
\end{array}\right\|
$$

There is no loss of generality in assuming, as indicated in the conditions, that the singular point $P$ is at the origin in the $u v w$-space, and that the transform of $P$ by (1) is the origin $P_{1}$ in the $x y z$-space. Neither will generality be lost if we assume for convenience that the determinant

$$
H_{1} \equiv\left|\begin{array}{lll}
J_{u} & J_{v} & J_{w} \\
\psi_{u} & \psi_{v} & \psi_{w} \\
\chi_{u} & \chi_{v} & \chi_{w}
\end{array}\right|
$$

is that one of the matrix (2) which does not vanish at the origin.

By our assumptions $(b)$ and $(c)$ the equations

$$
J(u, v, w)=0, \quad y=\psi(u, v, w), \quad z=\chi(u, v, w)
$$

have the initial solution $(u, v, w, y, z)=(0,0,0,0,0)$. The hypothesis $(d)$ justifies the assumption that the determinant (3) is different from zero, as we have seen. Hence by the usual theorems of implicit functions there exists a neighborhood $(0,0,0,0,0)_{\epsilon}{ }^{*}$ in which no two solutions $(u, v, w, y, z)$ of equations (4) have the same projection $(y, z)$, and a neighborhood $(0,0)_{\delta}$ of the point $(y, z)=(0,0)$ in which equations (4) determine $u, v, w$ as functions of class $C^{\prime \prime}$ of $y$ and $z$,

$$
u=u(y, z), \quad v=v(y, z), \quad w=w(y, z)
$$

* For these theorems see Bliss, Princeton Colloquium Lectures, pp. \&-9. By the notation $(0,0,0,0,0)_{\epsilon}$ is meant a neighborhood

$$
|u|<\epsilon, \quad|v|<\epsilon, \quad|w|<\epsilon, \quad|y|<\epsilon, \quad|z|<\epsilon
$$

of the point $(0,0,0,0,0)$. 
defining values $(u, v, w, y, z)$ in the neighborhood $(0,0,0,0,0)_{\mathrm{e}}$. By substituting these results in the third of equations (1), a surface

$$
x=X(y, z)
$$

is found, which is the transform by (1) of the surface $S$.

\section{The Envelope Curve $d_{1}$.}

We now interpret equations (1) as a two-parameter family of curves with the parameters $v, w$. Under the assumption $(d)$, the surface $S_{1}$ is the envelope of the curves (1).* If a one-parameter family of curves be chosen from the set (1), this family will not in general have an enveloping curve. The condition that a curve

$\left(d_{1}\right) \quad x=x(\alpha)=X[y(\alpha), z(\alpha)], \quad y=y(\alpha), \quad z=z(\alpha)$

on the surface $S_{1}$ shall be an envelope may be derived as follows. ${ }^{+}$

If we substitute $y(\alpha), z(\alpha)$ in the functions $v(y, z), w(y, z)$ defined by equations (5) two functions $v(\alpha), w(\alpha)$ are determined and a one-parameter family of curves is defined when $v(\alpha), w(\alpha)$ are substituted in (1). These curves are tangent to the curve $\left(d_{1}\right)$ if $y$ and $z$ are determined as functions of $\alpha$ so that

$$
x_{a}=\phi_{y} y_{a}+\phi_{z} z_{\alpha}=m \phi_{u}, \quad y_{a}=m \psi_{u}, \quad z_{\alpha}=m \chi_{u},
$$

$u$, $v$, and $w$ being thought of as functions of $y$ and $z$. The three determinants of the matrix

$$
\left\|\begin{array}{ccc}
\phi_{y} y_{a}+\phi_{z} z_{a} & y_{a} & z_{a} \\
\phi_{u} & \psi_{u} & \chi_{u}
\end{array}\right\|
$$

must therefore be zero, $i . e$., the three equations

$$
\begin{aligned}
\left(\phi_{u}-\phi_{y} \psi_{u}\right) y_{a}-\psi_{u} \phi_{z} z_{a} & =0 \\
\chi_{u} \phi_{y} y_{a}-\left(\phi_{u}-\chi_{u} \phi_{z}\right) z_{a} & =0 \\
\chi_{u} y_{a}-\psi_{u} z_{a} & =0,
\end{aligned}
$$

*W. V. Lovitt, "A type of singular points for a transformation of three variables," Transactions, vol. 16 (1915), p. 377.

+ Mason-Bliss, "The properties of curves in space which minimize a definite integral," Transactions, vol. 9 (1908), pp. 440-466. 
must be satisfied. The coefficients of $y_{a}, z_{a}$ in these equations cannot all vanish since at least one of the derivatives $\phi_{u}, \psi_{u}$, $\chi_{u}$ is different from zero at the point $P_{1}$. That any two of the equations are a consequence of the third may be shown by expanding the determinant of any pair of the equations and using the relation

$$
\phi_{u}-x_{y} \psi_{u}-x_{z} \chi_{u}=0 .
$$

The determination of a one-parameter family of curves having an enveloping curve $\left(d_{1}\right)$ is therefore to be effected by solving one of the above equations. It has the form

$$
A(y, z) y_{\alpha}+B(y, z) z_{\alpha}=0,
$$

when $u, v$, and $w$ are replaced by their values in terms of $y$ and $z$ from equations (5). Since this differential equation is of the first order there exists one and only one integral curve

$$
y=y(\alpha), \quad z=z(\alpha)
$$

in the $y z$-plane, passing through the point $y=z=0$ for $\alpha=0$. The equations of the family of curves tangent to $\left(d_{1}\right)$ are found by substituting $y(\alpha), z(\alpha)$ in the expressions for $v$ and $w$ in terms of $y$ and $z$ from equations (5) and then putting the resulting functions $v(\alpha), w(\alpha)$ in equations (1). A family of extremals

$$
x=\phi(u, \alpha), \quad y=\psi(u, \alpha), \quad z=\chi(u, \alpha)
$$

is thus found, which are tangent to $\left(d_{1}\right)$ when $u=u(\alpha)$. The equation of the envelope $\left(d_{1}\right)$ will then be

$$
x=\phi[u(\alpha), \alpha], \quad y=\psi[u(\alpha), \alpha], \quad z=\chi[u(\alpha), \alpha] .
$$

We have then the following theorem:

Theorem 1: Given a family of curves

$$
x=\phi(u, v, w), \quad y=\psi(u, v, w), \quad z=\chi(u, v, w),
$$

if on a particular curve $C_{1}$ the determinant $J$ vanishes at the point $P_{1}$ and one at least of the determinants of the matrix (2) is different from zero at $P_{1}$, then the family of curves has an enveloping surface $S_{1}$ which touches $C_{1}$ at $P_{1}$ and for which $P_{1}$ is not a singular point. On the surface $S_{1}$ there exists a unique curve $\left(d_{1}\right)$ without singular points, which passes through the point $P_{1}$ and envelopes a one-parameter family of curves, con- 
taining the curve $C_{1}$, which are the transforms by (1) of the lines, in the uvw-space, parallel to the u-axis.

\section{The Plane of Directions which Transforms into the} Direction of $d_{1}$.

Urner* has shown that the necessary and sufficient condition that two non-tangent curves through $P$ which have their directions distinct from the critical direction be rendered tangent by the transformation, is that the plane of their tangents at the point contain the line having the critical direction. Furthermore each plane of directions through the critical direction is compressed into a single direction. We ask, what is the plane of directions which is compressed into the direction of the envelope $\left(d_{1}\right)$ at $P_{1}$ ?

This plane must contain the line of critical direction

$$
\frac{u}{I_{1}}=\frac{v}{I_{2}}=\frac{w}{I_{3}} .
$$

It must contain the line

$$
v=w=0 .
$$

Thus the plane is completely determined, unless these directions coincide; but this cannot, in general, happen.
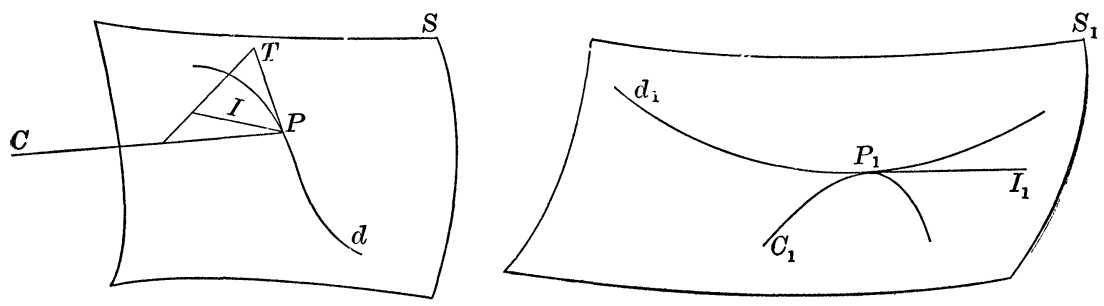

Designate by $\lambda$ the plane determined by $C$ and $I$. Every direction in $\lambda$ except $I$ goes into the same direction $I_{1}$ in the $x y z$-space. In particular the direction $T$ which is the intersection of $\lambda$ with the tangent plane to the surface $S$ at $P$ goes into the direction $I_{1}$. To the curve $\left(d_{1}\right)$ on the surface $S_{1}$ there corresponds a unique curve $(d)$ on the surface $S$ and from

* S. E. Urner, "Certain singularities of point transformations in space of three dimensions," Transactions, vol. 13 (1912), pp. 232-264. 
what we have just shown the curve $(d)$ must be tangent to the plane $\lambda$ at the point $P$.

We determine the differential equation for the curve $(d)$ as follows: obtain the equation of the plane $\lambda$. It is

$$
I_{3} v-I_{2} w=0 .
$$

The line $T$ is the intersection of this plane with the tangent plane to the surface $S$ at the point $P$. The projection of its direction on the $v w$-plane is

$$
I_{2} d w-I_{3} d v=0 .
$$

The integral curve of this equation which passes through the point $P$ is the projection of the curve $(d)$ upon the $v w$-plane. This integral together with the surface $S$ completely determines the curve $(d)$.

We have then the following theorem:

Theorem 2. The plane of directions in the uvw-space determined by the critical direction $I\left(I_{1}: I_{2}: I_{3}\right)$ and the line $v=w=0$ and containing the tangent line $T$ to the curve $(d)$ transforms into a single direction $I_{1}$ in the xyz-space. The direction $I_{1}$ is the direction of the tangent to the curve $\left(d_{1}\right)$, which curve is the transform of the curve (d) by means of equations (1).

\section{Illustration.}

The transformation

$$
x=u^{2}, \quad y=u+v, \quad z=v+w
$$

has for its jacobian $J \equiv 2 u$. Thus the jacobian surface $S$ is the surface $u=0$. Substitution in equation (3) gives us $H_{1} \equiv 2 \neq 0$. The critical direction is given by $1: 1: 1$. The line $(d)$ on $S$ and through $P\left(0, v_{0}, w_{0}\right)$ which transforms into $\left(d_{1}\right)$ on $S_{1}$ is the line

$$
v+w=v_{0}+w_{0}, \quad u=0 .
$$

The transform of $(d)$ is the line

$$
z=v_{0}+w_{0}, \quad x=0 .
$$

The surface $S_{1}$ is given by $x=0$. The lines

$$
v=c_{1}, \quad w=c_{2}, \quad u=t,
$$


which are parallel to the $u$-axis, are transformed into

$$
x=\left(y-c_{1}\right)^{2}, \quad z=c_{1}+c_{2},
$$

which are seen to be tangent to the surface $S_{1}$. It is evident from the last equations that those one-parameter families of parabolas which lie in the planes parallel to the $x y$-plane have envelopes, and that no others have. These envelopes are the curves $\left(d_{1}\right)$.

Purdue University.

\section{AN ELEMENTARY BOUNDARY VALUE PROBLEM.}

BY PROFESSOR DUNHAM JACKSON.

(Read before the American Mathematical Society, April 29, 1916.)

IT is intuitively obvious that if a simple continuous curve is given in the $(x, y)$-plane, and a continuous distribution of values along the curve, there will exist functions of $x$ and $y$ which are continuous in both variables together, and which take on the prescribed values along the curve. It is the purpose of the present note to give an analytic proof of this fact, by elementary means, and, in particular, without reference to potential theory.* The problem will be treated first for the case of a rectifiable curve, then for an arbitrary Jordan curve.

Let the equations

$$
x=f(s), \quad y=\varphi(s), \quad(0 \leqq s \leqq l),
$$

define a simple closed rectifiable curve $C$, the variable $s$ standing for the length of arc, and $l$ for the total length of the curve. It is assumed that the functions $f(s)$ and $\varphi(s)$ are continuous throughout their interval of definition, and that $f(0)=f(l), \varphi(0)=\varphi(l)$, but that with this exception no one pair of values $(x, y)$ is given by two distinct values of $s$. Let $F(s)$ be an arbitrary continuous function defined throughout the same interval, subject to the condition that $F(0)=F(l)$.

* I understand that Mr. R. E. Gleason has had occasion to deal with a similar problem in connection with a paper recently presented to the Society; see Bulletin, vol. 22 (1916), pp. 278-279. 